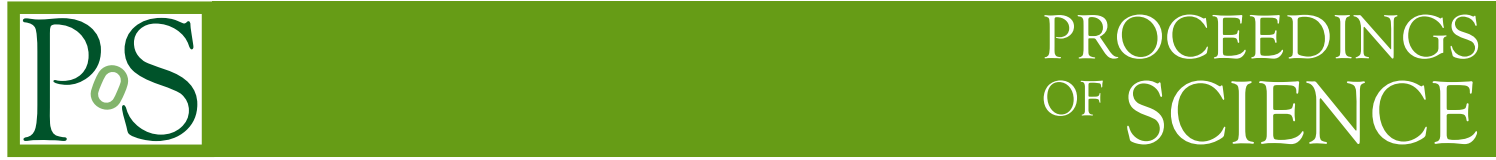

\title{
Interpretation of the top-quark mass measurements: a theory overview
}

\author{
Gennaro Corcella* \\ INFN, Laboratori Nazionali di Frascati \\ Via E. Fermi 40, I-00044 Frascati (RM), Italy \\ E-mail: gennaro.corcella@lnf.infn.it
}

I discuss the theoretical interpretation of the top-quark mass, which is extracted in standard and alternative measurements at the LHC. In particular, I point out that the top mass extracted in analyses relying on the use of Monte Carlo event generators must be close to the pole mass and review recent work aiming at estimating the theoretical uncertainty.

8th International Workshop on Top Quark Physics

14-18 September, 2015

Ischia, Italy

\footnotetext{
* Speaker.
} 


\section{Introduction}

The top quark mass is a fundamental parameter of the Standard Model, which, together with the $W$ mass, constrained the Higgs mass even before its discovery. It also plays a role in the result that the Standard Model vacuum lies on the border between stability and metastability regions [1], which relies on the assumption that the world average, $m_{t}=[173.34 \pm 0.27$ (stat) \pm 0.71 (syst) $] \mathrm{GeV}$ [2], is the top pole mass. Any deviation from this statement may change the conclusions of [1].

Top-quark mass measurements are performed by comparing experimental results with theory predictions: the extracted mass must thus be interpreted as the mass used in the calculation. Standard measurements, based on the template, matrix-element and ideogram methods (see, e.g., the recent analyses in [3,4]), rely on parton shower generators, such as HERWIG [5] or PYTHIA [6], which simulate the hard scattering at leading order (LO), multiple radiation in the soft or collinear approximation and are provided with models for hadronization. The aMC@NLO [7] and POWHEG [8] codes generate the hard process at NLO and are matched to HERWIG or PYTHIA for showers and hadronization. In principle, any theoretically well-defined top mass extraction would need at least a NLO calculation for top-quark production and decay, including interference effects: much debate has hence been taking place on whether measurements using parton showers and hadronization models correspond to any top-mass definition. As will be discussed hereinafter, the measured top mass must be close to the pole mass and work has been lately undertaken to assess the theoretical uncertainty. So-called alternative methods use other observables, such as total cross sections or distribution endpoints, which can be compared directly with fixed-order and possibly resummed QCD calculations, thus allowing a straightforward interpretation of the extracted mass.

In Section 2, I briefly review the main mass definitions; in Sections 3 I discuss in more detail the interpretation of the top mass results; in Section 4 I make some final remarks.

\section{Top mass definitions}

Mass definitions are related to the subtraction of the ultraviolet divergences in the renormalized self energy $\Sigma^{R}$, which, in dimensional regularization, with $d=4-2 \varepsilon$, at one loop in QCD, reads:

$\Sigma^{R} \simeq \frac{i \alpha_{S}}{4 \pi}\left\{\left(\frac{1}{\varepsilon}-\gamma+\ln 4 \pi+A\right) \not p-\left[4\left(\frac{1}{\varepsilon}-\gamma+\ln 4 \pi\right)+B\right] m_{0}\right\}+i\left[\left(Z_{2}-1\right) \not p-\left(Z_{2} Z_{m}-1\right) m_{0}\right]$,

where $m_{0}$ is the bare mass, $Z_{2}$ and $Z_{m}$ the wave-function and mass renormalization constants. The on-shell renormalization scheme, leading to the pole mass definition, is defined so that $\Sigma^{R}=0$ and $\partial \Sigma^{R} / \partial \not p=0$ for $\not p=0$; the $\overline{\mathrm{MS}}$ scheme fixes $Z_{2}$ and $Z_{m}$ in order to subtract the contributions $\sim \frac{1}{\varepsilon}-\gamma+\ln 4 \pi$ in Eq. (2.1). The renormalized propagators $S^{R}$ in the on-shell (o.s.) and $\overline{\mathrm{MS}}$ schemes are then expressed in terms of pole and $\overline{\mathrm{MS}}$ masses as follows:

$$
S_{\mathrm{o} . \mathrm{s} .}^{R}(p) \simeq \frac{i}{\not p-m_{\mathrm{pole}}}, S_{\overline{\mathrm{MS}}}^{R} \simeq \frac{i}{\not p-m_{\overline{\mathrm{MS}}}-(A-B) m_{\overline{\mathrm{MS}}}} .
$$

In Eq. (2.2) $m_{\text {pole }}$ is the pole of the propagator after renormalization, which is in agreement with the intuitive notion of the mass of a particle, whereas $m_{\overline{\mathrm{MS}}}$ may be quite far from the pole. The relation between pole and $\overline{\mathrm{MS}}$ masses was calculated up to four loops [9] and reads, for top quarks:

$$
m_{t, \text { pole }}=\bar{m}_{t}\left(\bar{m}_{t}\right)\left[1+0.42 \alpha_{S}+0.83 \alpha_{S}^{2}+2.37 \alpha_{S}^{3}+(8.49 \pm 0.25) \alpha_{S}^{4}+\mathscr{O}\left(\alpha_{S}^{5}\right)\right] .
$$


The last term in (2.3) yields an uncertainty of about $200 \mathrm{MeV}$ on the pole- $\overline{\mathrm{MS}}$ mass relation [9].

The self energy, when expressed in terms of the pole mass, is affected by infrared renormalons [10], i.e. the coefficients of $\alpha_{S}^{n}$ grow factorially:

$$
\Sigma^{R}\left(m_{\text {pole }}, m_{\text {pole }}\right) \approx m_{\text {pole }} \sum_{n} \alpha_{S}^{n+1}\left(2 b_{0}\right)^{n} n !
$$

Due to Eq. (2.4), the pole mass gets corrections $\Delta m_{\text {pole }} \simeq \mathscr{O}\left(\Lambda_{\mathrm{QCD}}\right)$, the so-called renormalon ambiguity. The $\overline{\mathrm{MS}}$ mass is renormalon-free, but it is not a threshold mass, as it exhibits corrections $\left(\alpha_{S} / v\right)^{k}, v$ being the top velocity, that are quite large for small $v$.

By using the recent computation [9] to fit the $\mathscr{O}\left(\alpha_{S}^{4}\right)$ coefficient of the renormalon calculation in [10] and extrapolating the result to predict also the higher-order terms, one can find that the renormalon ambiguity on the pole mass is even below $100 \mathrm{MeV}$ [11]. This result, along with the good convergence of the expansion (2.3), makes the top-quark pole mass a reliable quantity.

The MSR mass [12] depends on a parameter $R$, which could be, e.g., a factorization scale, and tries to interpolate between pole and $\overline{\mathrm{MS}}$ masses, i.e. $m^{\mathrm{MSR}}(R) \rightarrow m_{\text {pole }}$ for $R \rightarrow 0$ and $m^{\mathrm{MSR}}(R) \rightarrow$ $\bar{m}(\bar{m})$ for $R \rightarrow \bar{m}(\bar{m})$. Pole and MSR masses differ by a counterterm, i.e. $m_{\text {pole }}=m^{\mathrm{MSR}}(R, \mu)+$ $\delta m(R, \mu)$, where the $\mu$-dependence of $m^{\mathrm{MSR}}(R, \mu)$ follows renormalization group equations. The MSR mass is typically employed in the context of Soft Collinear Effective Theories (SCET).

\section{Interpretation of the experimental results}

Standard experimental measurements are carried out by using Monte Carlo simulations: since parton showers are not exact QCD calculations, the interpretation of the measured mass in terms of any field-theory mass definition is not straightforward and one often calls it 'Monte Carlo mass'. However, since these measurements are done by reconstructing top-decay $(t \rightarrow b W)$ observables, with on-shell top quarks, the extracted mass must mimic the pole mass, which is, by definition, the pole of the renormalized propagator. Such a simple picture is spoiled by the lack of higher-order corrections, as standard parton showers are matched to the tree-level matrix element [14] and do not fully contain one-loop and width $\left(\Gamma_{t}\right)$ effects, or by colour-reconnection effects. In fact, in the Monte Carlo hadronization models, it may happen that, for few events, the $b$ quark in top decay forms a $B$ meson with an antiquark from the initial state. Much work has therefore been undertaken to estimate the uncertainty on the identification of the measured mass with the pole mass.

As for NLO corrections, in the aMC@NLO code NLO top decays are implemented for singletop events [15]; in $t \bar{t}$ production, the decays are on shell, but spin correlations and part of the off-shell contributions are included via MadSpin [16]. In POWHEG, NLO top decays have been lately implemented [17], accounting for $\Gamma_{t}$ effects in different approximations.

As far as colour reconnection is concerned, Ref. [13] investigates it in the framework of the Lund string model, tuned to charged-particle multiplicity or transverse momentum data. It is found that the treatment of colour reconnection can lead to an uncertainty on the top mass within 200 and $500 \mathrm{MeV}$, according to the model which is chosen. Colour connection in the HERWIG cluster model was instead tackled in [18], where top quarks were forced to hadronize in top mesons and decay according to the spectator model. In this way, top quarks must form colour singlets with light quarks; also, by using lattice-based methods, Heavy Quark Effective Theory or Non Relativistic 

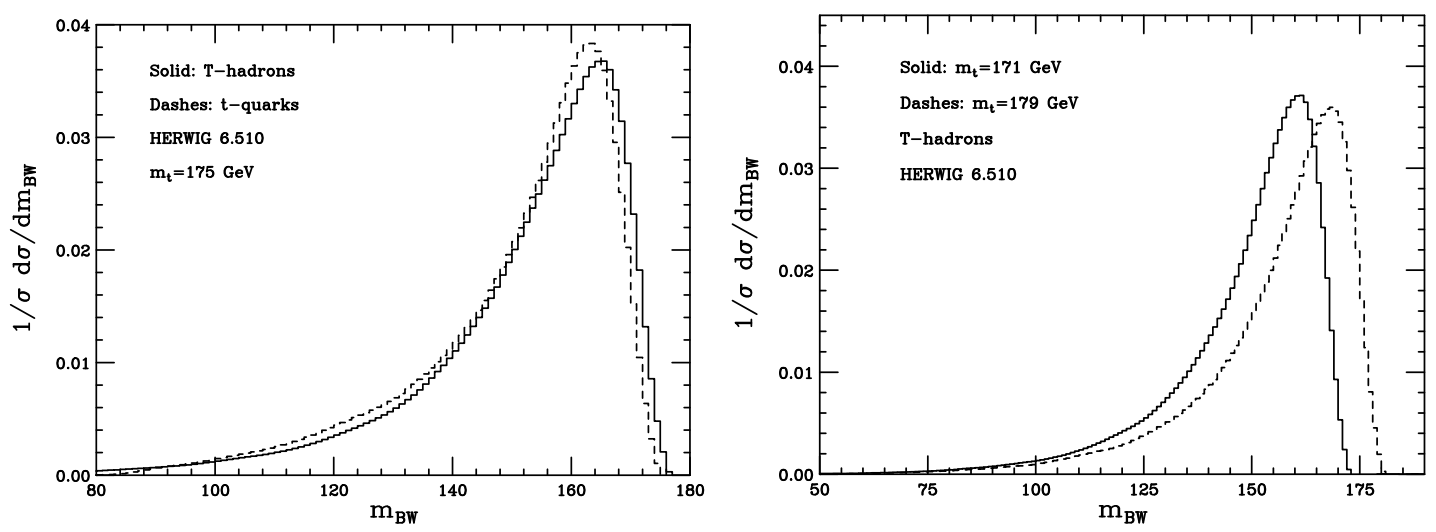

Figure 1: $B W$ invariant mass in $e^{+} e^{-} \rightarrow t \bar{t}$ events at $1 \mathrm{TeV}$. Left: comparison for $T$-hadron (solid) and standard top events (dashes) with $m_{t}=175 \mathrm{GeV}$. Right: $m_{B W}$ for $T$ hadrons and different values of $m_{t}$.

QCD, one can precisely connect a meson mass to a well-defined quark-mass definition. Such a study does not aim at detecting $T$-hadrons, but rather, by comparing observables in standard $t \bar{t}$ samples and in $T$-hadron events, it may shed light on the non-perturbative uncertainty on $m_{t}$. In Fig. 1 I present the $B W$ invariant mass distribution, $B$ being a $b$-flavoured hadron in top decay, for $e^{+} e^{-} \rightarrow t \bar{t}$ collisions at $1 \mathrm{TeV}$, in the dilepton channel, by using the HERWIG 6 event generator. If top quarks hadronize before decaying, $m_{B W}$ is shifted towards higher values (Fig. 1, left), with respect to standard top decays; in fact, in $T$ decays, the $b$ quark likely forms with the spectator quark a cluster of small invariant mass, decaying into a $B$ meson, plus soft hadrons, e.g. pions. Therefore, $m_{B W}$ tends to be closer to the kinematic limit, given by the mass of the $T$-hadron. Figure 1 (right) presents the $m_{B W}$ just for $T$ hadrons and $m_{t}=171$ and $179 \mathrm{GeV}$. Work is in progress to quantify the discrepancies in Fig. 1 in terms of an uncertainty on $m_{t}$ and its interpretation as a pole mass.

In the SCET framework [12] the jet mass plays the role of a MSR mass, with $R=\Gamma_{t}$ in the case of $e^{+} e^{-} \rightarrow t \bar{t}$ events, and can be related at NLO to the pole mass as follows:

$$
m_{J}(\mu)=m_{\text {pole }}-\frac{\alpha_{S}(\mu) C_{F} \Gamma_{t}}{\pi}\left(\ln \frac{\mu}{\Gamma_{t}}+\frac{3}{2}\right)+\mathscr{O}\left(\alpha_{S}^{2}\right) .
$$

One then assumes [19] that the measured $m_{t}$ is a jet mass at the scale of shower cutoff $Q_{0} \simeq 1 \mathrm{GeV}$, yielding an uncertainty $m_{\text {pole }}-m_{J}\left(Q_{0}\right) \simeq 200 \mathrm{MeV}$. Within SCET, one may compare resummed distributions, such as the thrust in $e^{+} e^{-} \rightarrow t \bar{t}$, using the MSR mass for $R \sim \mathscr{O}(1 \mathrm{GeV})$, with Monte Carlo spectra and calibrate the Monte Carlo mass to reproduce the SCET prediction [20].

Another approach was suggested in [21]: one first performs a simultaneous fit of the Monte Carlo mass and of a given observable, such as total or differential cross sections, and then compares with a (N)NLO calculation for the same quantity, using, e.g., the pole mass. The conclusion of Ref. [21] is that the uncertainty on this calibration is roughly $2 \mathrm{GeV}$.

As for the alternative measurements, a typical example is the total $t \bar{t}$ cross section, which was calculated to NNLO+NNLL accuracy in [22], and allows a direct extraction of the pole mass $[23,24]: m_{t}=\left(172.9_{-2.6}^{+2.5}\right) \mathrm{GeV}$ (ATLAS) and $m_{t}=\left(173.6_{-1.8}^{+1.7}\right) \mathrm{GeV}(\mathrm{CMS})$, combining 7 and 8 $\mathrm{TeV}$ data. In principle, even this extraction depends on the use of event generators to evaluate the acceptance, but nevertheless both ATLAS and CMS found a mild dependence on the mass implemented in the Monte Carlo code. Reference [25] investigated NNLO total and NLO differential 
cross sections in terms of the $\overline{\mathrm{MS}}$ mass and obtained an overall milder scale dependence; the recent calculation of NNLO differential spectra [26] should shade more light on this finding.

The top pole mass was also determined from the $t \bar{t}+1$ jet cross section, following [27], which has a stronger dependence on $m_{t}$ with respect to the inclusive cross section. ATLAS performed this measurement by using POWHEG, matched to PYTHIA, taking care of unfolding detector, hadronization and shower effects, in such a way to recover the partonic $t \bar{t} j$ result. The result is $m_{t, \text { pole }}=\left[173.7 \pm 1.5\right.$ (stat.) \pm 1.4 (syst.) ${ }_{-0.5}^{+1.0}$ (th.) $] \mathrm{GeV}$; the impact of the Monte Carlo input mass in the evaluation of the acceptance is negligible.

Other observables, which have been lately proposed, are the peak of the $b$-jet energy spectrum, the $b$-jet+lepton invariant mass $m_{b \ell}$ and a few distribution endpoints. The general feature of these measurements is that they rely on the kinematic reconstruction of top-decay final states and hence, once again, the extracted mass must be close to the pole mass. In detail, the $b$-jet energy measurement [29] exhibits the property [30] that the position of the peak is independent of the boost from the top to the laboratory frame, as well as of the production mechanism. The experimental measurement yields $m_{t}=[172.29 \pm 1.17$ (stat.) \pm 2.66 (syst.) $] \mathrm{GeV}$ at $8 \mathrm{TeV}$ : however, the invariance of the peak position is only valid at LO and for inclusive spectra, and therefore it will be interesting updating the analysis [29] by using NLO top decays.

The $m_{b \ell}$ spectrum was used by CMS to reconstruct $m_{t}$ in the dilepton channel: by comparing with the MadGraph+PYTHIA (LO) simulation, $m_{t}=(172.3 \pm 1.3) \mathrm{GeV}$ was found [31]. Nevertheless, the NLO calculation of $m_{b l}$ [32], employing the pole mass, is available and exhibits some discrepancies with respect to LO parton showers $[18,33]$ : an extension to NLO is thus mandatory.

Reference [34] measures $m_{t}$ from the endpoints of the $m_{b \ell}, \mu_{b b}$ and $\mu_{\ell \ell}$ spectra, where $\mu_{b b}$ and $\mu_{\ell \ell}$ are related to the $b b$ and $\ell \ell$ invariant masses. This analysis minimizes the Monte Carlo systematics, since the $b$-jet can be calibrated directly from data; the leftover Monte Carlo uncertainties are mostly due to the assumption that the $t$ and $\bar{t}$ decay chains are independent of colour reconnection. The result, based on LO kinematic relations, is $m_{t}=[173.9 \pm 0.9 \text { (stat. })_{-2.1}^{+1.7}$ (syst.) $] \mathrm{GeV}$; comparing the data with the recent implementation of POWHEG [17], accounting for some interference effects, can therefore give some insight on the uncertainty due to higher-order corrections.

\section{Conclusions}

I discussed the interpretation of the top mass results at the LHC: measurements relying on the reconstruction of top-decay products yield results close to the top-quark pole mass, with an uncertainty due to the approximations in the computations used for comparison, namely missing loop and width corrections and colour-reconnection effects. Using the recent calculation of the relation between pole and $\overline{\mathrm{MS}}$ masses, the renormalon ambiguity can be proved to be smaller than $100 \mathrm{MeV}$, thus making the pole mass a suitable quantity. Work has been done to quantify the uncertainty on the interpretation of the measured mass as a pole mass, by using Soft Collinear Effective Theories or simulating fictitious top-hadron states. Alternative measurements, based on the extraction from the cross sections of $t \bar{t}$ and $t \bar{t} j$ events, yield the pole mass, up to small acceptance and hadronization corrections. Other strategies, using energy peaks, endpoints and $m_{b \ell}$ look promising and worthwhile to be pursued at $13 \mathrm{TeV}$, thanks to the higher statistics and the late implementation of NLO top decays in shower generators. 


\section{References}

[1] G. Degrassi, S. Di Vita, J. Elias-Miro, J. R. Espinosa, G. F. Giudice, G. Isidori and A. Strumia, JHEP 1208 (2012) 098.

[2] ATLAS and CDF and CMS and D0 Collaborations, arXiv:1403.4427 [hep-ex].

[3] ATLAS Collaboration, Eur. Phys. J. C75 (2015) 158.

[4] CMS Collaboration, arXiv:1509.04044 [hep-ex].

[5] G. Corcella et al., JHEP 0101 (2001) 010.

[6] T. Sjöstrand, S. Mrenna and P.Z. Skands, JHEP 0605 (2006) 026.

[7] J. Alwall et al., JHEP 1407 (2014) 079.

[8] S. Alioli, P. Nason, C. Oleari and E. Re, JHEP 1006 (2010).

[9] P. Marquard, A.V. Smirnov, V.A. Smirnov and M. Steinhauser, Phys. Rev. Lett. 114 (2015) 142002.

[10] M. Beneke and V.N. Braun, Nucl. Phys. B426 (1994) 301.

[11] P. Nason, arXiv:1602.00443 [hep-ph].

[12] S. Fleming, A.H. Hoang, S. Mantry, I.W. Stewart, Phys. Rev. D77 (2008) 114003.

[13] S. Argyropoulos and T. Sjöstrand, JHEP 1411 (2014) 043.

[14] G. Corcella and M.H. Seymour, Phys. Lett. B442 (1998) 417.

[15] A.S. Papanastasiou, R. Frederix, S. Frixione, V. Hirschi and F. Maltoni, Phys. Lett. B726 (2013) 223.

[16] P. Artoisenet, R. Frederix, O. Mattelaer and R. Rietkerk, JHEP 1303 (2013) 015.

[17] J.M. Campbell, R.K. Ellis, P. Nason and E. Re, JHEP 1504 (2015) 114.

[18] G. Corcella, EPJ Web Conf. 80 (2014) 00019.

[19] A.H. Hoang and I.W. Stewart, Nucl. Phys. Proc. Suppl. 185 (2008) 220.

[20] A.H. Hoang, arXiv:1412.3649 [hep-ph].

[21] J. Kieseler, K. Lipka and S.-O. Moch, arXiv:1511.00841 [hep-ph].

[22] M. Czakon, P. Fiedler and A.D. Mitov, Phys. Rev. Lett. 110 (2013) 252004.

[23] CMS Collaboration, CMS-PAS-TOP-13-004.

[24] ATLAS Collaboration, Eur. Phys. J. C74 (2015) 3109.

[25] M. Dowling and S.-O. Moch, Eur. Phys. J. C74 (2014) 3167.

[26] M. Czakon, D. Heymes and A.D. Mitov, arXiv:1511.00549 [hep-ph].

[27] S. Alioli, P. Fernandez, J. Fuster, A. Irles S.-O. Moch, P. Uwer and M. Vos, Eur. Phys. J. C73 (2013) 2438.

[28] ATLAS Collaboration, JHEP 1510 (2015) 121.

[29] CMS Collaboration, CMS-PAS-TOP-15-002.

[30] K. Agashe, R. Franceschini and D. Kim, Phys. Rev. D88 (2013) 057701.

[31] CMS Collaboration, CMS-PAS-TOP-14-014.

[32] S. Biswas, K. Melnikov and M. Schulze, JHEP 1008 (2010) 048.

[33] G. Corcella and F. Mescia, Eur. Phys. J. C65 (2010) 171; Erratum-ibid. C68 (2010) 687.

[34] CMS Collaboration, Eur. Phys. J. C73 (2013) 2494. 